\title{
How to find the most practical ecosystem management plan
}

\author{
T. C. Haas \\ Lubar School of Business Administration, \\ University of Wisconsin at Milwaukee, USA
}

\begin{abstract}
A predictive understanding of how political processes produce a sequence of ecosystem management decisions would allow environmental managers to estimate how much social change would be needed to make an ecosystem sustainable. To this end, a stochastic, temporal model of how political processes influence and are influenced by ecosystem processes is being developed. This model is realized in a set of interacting Influence Diagrams (Bayes Nets with Decision nodes) that each represent the belief systems of political groups in countries that affect an ecosystem. These group models also interact with a model of the affected ecosystem.

After these models are fitted to a political-ecological data set, they are used to find the most practical ecosystem management plan by modifying the modeled group belief systems that were estimated from the data, until a sequence of group actions towards the ecosystem over a future time period results in desired ecosystem values at a designated future time point, e.g. a viable wildlife population in the year 2058. Belief systems are minimally modified away from their data-based values so as to produce this desired sequence of group actions towards the ecosystem.

Such a set of interacting models has been constructed for the management of the endangered Cheetah (Acinonyx jubatus) across Kenya, Tanzania, and Uganda. Presidential offices, wildlife protection agencies, rural residents, pastoralists, and NGO groups within these countries are modeled along with a model of the cheetahsupporting ecosystem. A data set has been collected that consists of political actions by these groups along with cheetah counts by political region. The most practical management plan for this case is computed.

Keywords: ecosystem management, environmental politics, wildlife management, biodiversity, optimal policies.
\end{abstract}




\section{Introduction}

Problem: many of the earth's species may be heading for extinction in habitats that are not under the political control of any one country. Those countries that share some of this habitat within their political boundaries, are often developing and have inadequate resources for wildlife conservation - while most of the world's resources for wildlife conservation are expended by developed countries on internal programs. With our current understanding of biological processes, this potential species loss is irreversible. Because of this irreversibility, it can be argued that this problem should be of high priority to all countries. This article gives one way to address this problem.

Because of the above-mentioned developed-country funding bias, lack of conservation funds in habitat-enclosing countries, and lack of political control over these habitats by any one country, conventional conservation programs such as command and control or adaptive management, may not be able to save a managed species from extinction. These considerations have motivated the development here of an approach to ecosystem management that does not assume central control but instead, after building models of both the political processes at work in the habitat-dominating countries and the dynamics of the species-supporting ecosystem, searches for politically feasible ecosystem management plans. Thus, ecosystem management is seen as a two-step procedure: first understand how the political-ecological process works at a mechanistic level and only then begin a search for management plans that require the least change in human behavior patterns in order to effect behavior changes that result in a sequence of actions that leads to the survival of the species being managed. Understanding political processes is seen to be critical because ultimately, the decision to implement ecosystem protection policies is a political one. Management plans that are suggested by examining the output of these ecosystem-only models that ignore political processes may not be supported by the responsible wildlife protection agencies or other affected social groups (hereafter, groups) unless the plan addresses the goals of each such group.

This two-step approach to ecosystem management has been implemented in a suite of integrated, web-based analysis tools called an Ecosystem Management Tool (EMT). Use of the EMT allows an analyst to link political processes and goals to ecosystem processes and desired ecosystem outcomes. The EMT's central component is a quantitative, stochastic, and causal model of the ecosystem being managed and the groups involved with this management. This model is called the political-ecological process simulator or simply simulator. This simulator expresses the group decision making models and the ecosystem model in probabilistic structures known as influence diagrams (IDs) (see Pearl [1]). The other components of the EMT are links to data streams, freely-available software for performing all ecosystem management computations and displays, and a webbased archive and delivery system for the first three of these components. The two main uses of the EMT are first to find practical ecosystem management plans, and second to allow anyone with web access to assess for themselves the status of 
a species being managed with the EMT. This second use is intended to make more accessible to developed countries the status and challenges of managing critical ecosystems in distant, developing countries.

To demonstrate its feasibility, a working EMT for the management of cheetah in a portion of east Africa is developed herein. The portion of east Africa studied is the land enclosed by the political boundaries of Kenya, Tanzania, and Uganda. This ecosystem involves the cheetah, their prey, their physical habitat, and those groups who directly and indirectly manage the cheetah population. Specifically, the simulator models (a) the president, environmental protection agency (EPA), nonpastoralist rural residents (hereafter, rural residents), and pastoralists of Kenya, Tanzania and Uganda; (b) a group of nongovernmental organizations (NGOs) that seek to protect biodiversity within these countries; and (c) the cheetah-supporting ecosystem enclosed by these countries.

Once the simulator has been fitted to a political-ecological data set, the simulator can be used to construct the most practical ecosystem management plan. In this article, a practical plan is one that demands the least change in group behavior patterns for a desired improvement in ecosystem health as measured by the ecosystem ID's output nodes. This definition emphasizes political feasibility over a plan's cost. Such a plan is referred to herein as the Most Practical Ecosystem Management Plan (MPEMP).

\section{Simulator function and parameter estimation}

\subsection{Simulator function}

The simulator functions by having each group implement an action chosen from a pre-determined repertoire that maximizes their multiobjective (multiple goals) objective function. These actions are in-turn, reacted to by the other groups and may also impact the ecosystem. A temporal sequence of actions taken by groups that affect the ecosystem (the result of playing this sequential game), is called an ecosystem management plan (EMP). Such an actions history may or may not be the result of a formal, articulated policy for managing the ecosystem. See Haas [2] and [3] for complete descriptions of all group IDs and the ecosystem ID. The model that emerges through the interactions of these IDs is referred to here as an Interacting IDs (IntIDs) model.

\subsection{The consistency analysis parameter estimator}

IntIDs model parameters are estimated via a procedure referred to here as Consistency Analysis (CA) described next. Let $\mathbf{U}^{(i)}$ be the vector that contains all of the chance nodes that make up the $i^{\text {th }}$ ID. IDs 1 through $m$ are models of group perceptions and decision making while the $(m+1)^{\text {th }}$ ID is the model of the ecosystem. Let $\boldsymbol{\beta}^{(G r p)}=\left(\boldsymbol{\beta}^{(1)^{\prime}}, \ldots, \boldsymbol{\beta}^{(m)^{\prime}}\right)^{\prime}$ be the stacked vector of group ID parameters wherein $\boldsymbol{\beta}^{(i)}$ parameterizes the $i^{\text {th }}$ group ID. Let $\boldsymbol{\beta}^{(E c o)}$ parameterize 
the ecosystem ID. Finally, let $\left.\boldsymbol{\beta}=\left(\boldsymbol{\beta}^{(G r p)}\right)^{\prime}, \boldsymbol{\beta}^{(E c o)^{\prime}}\right)^{\prime}$ parameterize the entire IntIDs model. Let $g_{S}^{(i)}\left(\boldsymbol{\beta}^{(i)}\right)$ be a goodness-of-fit statistic that measures the agreement of the $i^{\text {th }}$ ID's probability distribution (referred to here as the $\mathbf{U}^{(i)} \mid \boldsymbol{\beta}^{(i)}$ distribution) and the sample (data set), $S$. Larger values of $g_{S}^{(i)}\left(\boldsymbol{\beta}^{(i)}\right)$ indicate better agreement.

Each parameter in the model is assigned an a-priori value derived from either expert opinion, subject matter theory, or the results of a previous Consistency Analysis. Let $\beta_{H}^{(j)}$ be such a value assigned to the IntID's $j^{\text {th }}$ parameter. Collect all of these hypothesis parameter values into the hypothesis parameter vector, $\boldsymbol{\beta}_{H}$. Let $g_{H}^{(i)}\left(\boldsymbol{\beta}^{(i)}\right)$ be the agreement between the distribution identified by the values of $\boldsymbol{\beta}_{H}^{(i)}$ (the $\mathbf{U}^{(i)} \mid \boldsymbol{\beta}_{H}^{(i)}$ distribution) and the $\mathbf{U}^{(i)} \mid \boldsymbol{\beta}^{(i)}$ distribution. As with $g_{S}^{(i)}\left(\boldsymbol{\beta}^{(i)}\right)$, larger values of $g_{H}^{(i)}\left(\boldsymbol{\beta}^{(i)}\right)$ indicate better agreement. Note that $g_{S}^{(i)}\left(\boldsymbol{\beta}^{(i)}\right)$ is the agreement between a sample and a stochastic model, the $\mathbf{U}^{(i)} \mid \boldsymbol{\beta}^{(i)}$ distribution - while $g_{H}^{(i)}\left(\boldsymbol{\beta}^{(i)}\right)$ is the agreement between two stochastic models: the $\mathbf{U}^{(i)} \mid \boldsymbol{\beta}_{H}^{(i)}$ distribution and the $\mathbf{U}^{(i)} \mid \boldsymbol{\beta}^{(i)}$ distribution.

In what follows, the agreement between two distributions will be computed via the probability density probability function (PDPF). For a joint event, $\mathbf{u}^{(i)}$ described by an ID, this function is written as $p f_{\mathbf{U}^{(i)} \mid \boldsymbol{\beta}^{(i)}}\left(\mathbf{u}^{(i)}\right)$ (see Haas [4]). This function is a generalization of a probability mass function and a probability density function and is necessary because IDs that make up the political-ecological process simulator may contain a mixture of quantitative and qualitative chance nodes.

Let $\operatorname{gmax}_{S}^{(i)}$ be the unconstrained maximum value of $g_{S}^{(i)}\left(\boldsymbol{\beta}^{(i)}\right)$ over all $\boldsymbol{\beta}^{(i)}$. Similarly, let $\operatorname{gmax}_{H}^{(i)}$ be the unconstrained maximum value of $g_{H}^{(i)}\left(\boldsymbol{\beta}^{(i)}\right)$ over all $\boldsymbol{\beta}^{(i)}$. Up to errors in the approximation of $g_{H}^{(i)}\left(\boldsymbol{\beta}^{(i)}\right)$, this value is $g_{H}^{(i)}\left(\boldsymbol{\beta}_{H}^{(i)}\right)$. The Consistency Analysis parameter estimator maximizes the function

$$
g_{C A}^{(i)}\left(\boldsymbol{\beta}^{(i)}\right) \equiv\left(1-c_{H}\right)\left[\frac{g_{S}^{(i)}\left(\boldsymbol{\beta}^{(i)}\right)}{\left|\operatorname{gmax}_{S}^{(i)}\right|+1}\right]+c_{H}\left[\frac{g_{H}^{(i)}\left(\boldsymbol{\beta}^{(i)}\right)}{\left|\operatorname{gmax}_{H}^{(i)}\right|+1}\right]
$$

where $c_{H} \in(0,1)$ is the analyst's priority of having the estimated distribution agree with the hypothesis distribution as opposed to agreeing with the empirical (data-derived) distribution. Let $\boldsymbol{\beta}_{C}^{(i)} \equiv \operatorname{argmax}_{\boldsymbol{\beta}^{(i)}}\left\{g_{C A}^{(i)}\left(\boldsymbol{\beta}^{(i)}\right)\right\}$ be the Consistency Analysis estimate of $\boldsymbol{\beta}^{(i)}$. Hereafter, $\boldsymbol{\beta}_{C}^{(i)}$ will be referred to as the consistent parameter vector for the $i^{\text {th }}$ ID. See Haas [4] for further details on Consistency Analysis.

\subsection{Agreement functions}

\subsubsection{Data agreement functions}

Call a time series of action-actor-target observations an actions history data set. Let $g_{S}^{(G r p)}(\boldsymbol{\beta})$ be the agreement between the sequence of group actions produced by the IntIDs model and the actions history data set. Let $g_{S}^{(E c o)}(\boldsymbol{\beta})$ be the agreement 
between the time series of ecosystem mean values computed by the ecosystem ID and the observed time series of ecosystem values. For the entire IntIDs model, $g_{S}(\boldsymbol{\beta})=g_{S}^{(G r p)}(\boldsymbol{\beta})+g_{S}^{(E c o)}(\boldsymbol{\beta})$.

2.3.1.1 Agreement with Actions History Data The Overall Goal Attainment (OGA) node in a group ID represents the group's perceived utility. The actiontarget combination that maximizes the expected value of this node is the one that the group implements.

Let out ${ }_{i}^{(o b s)}\left(t_{j}\right)$ be group $i$ 's observed output action-target combination at time $t_{j}$; out ${ }_{i}^{(o p t)}\left(t_{j}\right)$ be the action-target combination computed by the group's ID at that time; and $M_{i j}$ be unity if out ${ }_{i}^{(o p t)}\left(t_{j}\right)=$ out $_{i}^{(o b s)}\left(t_{j}\right)$ and zero, otherwise. Let $\operatorname{EOGA}_{i, j}^{(o b s)}(\boldsymbol{\beta})=E\left[O G A^{(i)} \mid\right.$ out $\left._{i}^{(o b s)}\left(t_{j}\right)\right]$, and $\operatorname{EOGA}_{i, j}^{(o p t)}(\boldsymbol{\beta})=E\left[O G A^{(i)} \mid\right.$ out $\left._{i}^{(o p t)}\left(t_{j}\right)\right]$. Then $g_{S}^{(G r p)}\left(\boldsymbol{\beta}^{(G r p)}\right)=\sum_{i=1}^{m} g_{S}^{(i)}\left(\boldsymbol{\beta}^{(i)}\right)$ where

$$
\begin{aligned}
g_{S}^{(i)}(\boldsymbol{\beta}) \equiv & \sum_{j=1}^{T} M_{i, j}+\left(1-M_{i, j}\right)\left[\max \left\{\operatorname{EOGA}_{i, j}^{(o b s)}(\boldsymbol{\beta}), .001\right\}\right. \\
& \left.-\max \left\{\operatorname{EOGA}_{i, j}^{(o p t)}(\boldsymbol{\beta}), .001\right\}\right] .
\end{aligned}
$$

2.3.1.2 Agreement with ecosystem state data Say that a multivariate time series of ecosystem node values has been observed. For example, here, cheetah and herbivore counts are observed over time. Let $\mathbf{u}_{o b s}(t)$ be the vector of these values at time $t$. This vector constitutes a size-one sample on the observable ecosystem ID nodes at $t$. For such a sample, the negative Hellinger distance is $-\mid 1-\sqrt{p f_{\mathbf{U}^{(E c o)} \mid \boldsymbol{\beta}^{(E c o)}\left(\mathbf{u}_{o b s}(t)\right)}}$ (see Lindsay [5]). Let $g_{S}^{(E c o)}(\boldsymbol{\beta})$ be the sum of each of these negative Hellinger distances over each combination of region and time point.

\subsubsection{Hypothesis agreement function}

PDPF values of under an ID's hypothesis distribution, $\mathbf{U}^{(i)} \mid \boldsymbol{\beta}_{H}^{(i)}$ and its $\mathbf{U}^{(i)} \mid \boldsymbol{\beta}^{(i)}$ distribution are approximated by first drawing a size- $n$ sample of design points from a multivariate uniform distribution on the ID's chance nodes: $\mathbf{u}_{1}, \ldots, \mathbf{u}_{n}-$ and then estimating $p f_{\mathbf{U}^{(i)} \mid \boldsymbol{\beta}}^{(i)}\left(\mathbf{u}_{i}\right)$ with an $l$ nearest-neighbor, nonparametric density estimator due to Thompson and Tapia [6] at each of these design points. Using these estimates, the Hellinger distance between $\mathbf{U}^{(i)} \mid \boldsymbol{\beta}_{H}^{(i)}$ and $\mathbf{U}^{(i)} \mid \boldsymbol{\beta}^{(i)}$ is:

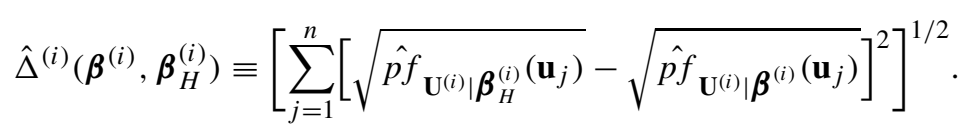

For the collection of group IDs, $g_{H}^{(G r p)}\left(\boldsymbol{\beta}^{(G r p)}\right)=\sum-\hat{\Delta}^{(i)}\left(\boldsymbol{\beta}^{(i)}, \boldsymbol{\beta}_{H}^{(i)}\right)$ where summation is over all combinations of time point, group and output node values 
considered by each group at each time point. For the ecosystem ID, $g_{H}^{(E c o)}(\boldsymbol{\beta})=$ $\sum-\hat{\Delta}^{(E c o)}\left(\boldsymbol{\beta}^{(E c o)}, \boldsymbol{\beta}_{H}^{(E c o)}\right)$ where summation is over all combinations of region and time point. Then, for the entire IntIDs model the measure of agreement with the hypothesis parameter values is $g_{H}(\boldsymbol{\beta})=g_{H}^{(G r p)}\left(\boldsymbol{\beta}^{(G r p)}\right)+g_{H}^{(E c o)}\left(\boldsymbol{\beta}^{(E c o)}\right)$.

\section{The MPEMP: definition and algorithm}

\subsection{Definition}

Let $\mathbf{Q}(\boldsymbol{\beta})$ be a vector of ecosystem state quantities that are modeled by the simulator's ecosystem ID. For example, $\mathbf{Q}$ (.) could be cheetah count and herbivore count in the year 2058. Assume that a Consistency Analysis has produced a set of consistent parameter values contained in $\boldsymbol{\beta}_{C}$ and the hypothesis parameter vector has been updated to these values, i.e., $\boldsymbol{\beta}_{H}=\boldsymbol{\beta}_{C}$. Using this updated $\boldsymbol{\beta}_{H}^{(G r p)}$, one way to quantify the concept of a practical ecosystem management plan described in the Introduction is to associate political feasibility with the value of $g_{H}^{(G r p)}\left(\boldsymbol{\beta}_{\text {MPEMP }}\right)$ where $\boldsymbol{\beta}_{\text {MPEMP }}$ is a set of group ID parameter values that have been modified away from this updated $\boldsymbol{\beta}_{H}^{(G r p)}$ so that a desired ecosystem state (expressed as a set of desired values for $\mathbf{Q}($.$) , namely \mathbf{q}$ ) is achieved by a sequence of group ID actions over a future time period. The idea is to find a set of minimal changes in group beliefs from those represented by $\boldsymbol{\beta}_{H}^{(G r p)}$ so that these groups change their behaviors enough to allow the ecosystem to respond in a desired manner. In other words, the MPEMP is the ecosystem management plan that emerges by finding group ID parameter values that result in a desired ecosystem state but that deviate minimally from $\boldsymbol{\beta}_{H}^{(G r p)}$. Formally, $\boldsymbol{\beta}_{\text {MPEMP }}=$ $\operatorname{argmax} \boldsymbol{\beta}^{(G r p)}\left\{g_{H}^{(G r p)}\left(\boldsymbol{\beta}^{(G r p)}\right)\right\}$ under the constraint that the ecosystem ID produces output values that are close to the desired ones, $\mathbf{q}$.

\subsection{Algorithm to find the MPEMP}

First, define an ecosystem damage utility function, $f_{\text {ecodam }}\left(\boldsymbol{\beta}^{(G r p)}\right)$ to be $\sum_{t, i, j}$ $E\left[O G A_{t}^{(i)} \mid\right.$ ecodam-action $\left._{i, j}\right]$ where $O G A_{t}^{(i)}$ is group $i$ 's Overall Goal Attainment node at time $t$, and ecodam-action $i, j$ is group $i$ 's $j^{\text {th }}$ ecosystem-damaging action. The sum is over all groups that directly affect the ecosystem, all ecosystemdamaging actions by these groups, and all time points at which such actions are executed during a run of the simulator. Then the MPEMP can be found with the following algorithm.

1. Perform a Consistency Analysis with the current $\boldsymbol{\beta}_{H}$ and a politicalecological data set to find $\boldsymbol{\beta}_{C}$. Update $\boldsymbol{\beta}_{H}$ to this $\boldsymbol{\beta}_{C}$.

2. Specify q, e.g. 2,000 cheetah and 10,000 herbivores in 2058 .

3. Set $k=1$ and iterate the following three steps: 
(a) Compute

$$
\boldsymbol{\beta}_{k}^{(G r p)}=\operatorname{argmin}_{\boldsymbol{\beta}^{(G r p)}}\left\{\left[\frac{f_{\text {ecodam }}\left(\boldsymbol{\beta}^{(G r p)}\right)}{f_{\text {ecodam }}\left(\boldsymbol{\beta}_{H}^{(G r p)}\right)}\right]+d(\boldsymbol{\beta})\right\}
$$

subject to

$$
\frac{\left|g_{H}^{(G r p)}\left(\boldsymbol{\beta}_{H}^{(G r p)}\right)-g_{H}^{(G r p)}\left(\boldsymbol{\beta}^{(G r p)}\right)\right|}{g_{H}^{(G r p)}\left(\boldsymbol{\beta}_{H}^{(G r p)}\right)}<0.1 k
$$

where $d(\boldsymbol{\beta})=\|E[\mathbf{Q}(\boldsymbol{\beta})]-\mathbf{q}\| /\left\|E\left[\mathbf{Q}\left(\boldsymbol{\beta}_{H}\right)\right]-\mathbf{q}\right\|$ and $\boldsymbol{\beta}=\left(\boldsymbol{\beta}^{(G r p)^{\prime}}\right.$, $\left.\boldsymbol{\beta}_{H}^{(E c o)^{\prime}}\right)^{\prime}$.

(b) If $d\left(\boldsymbol{\beta}_{k}\right)=0$ or $d\left(\boldsymbol{\beta}_{k-2}\right)=d\left(\boldsymbol{\beta}_{k-1}\right)=d\left(\boldsymbol{\beta}_{k}\right)$, set $\boldsymbol{\beta}_{\text {MPEMP }}$ to $\boldsymbol{\beta}_{k}^{(G r p)}$ and stop the iteration.

(c) Set $k=k+1$.

During the optimization, as ecosystem-damaging actions become less attractive to the group, they will not be executed and hence will not contribute to the sum that forms $f_{\text {ecodam }}\left(\boldsymbol{\beta}^{(G r p)}\right)$. Note that different sequences of group actions can lead to different values in the vector $E[\mathbf{Q}(\boldsymbol{\beta})]$. The utility of such an action needs to be computed under relevant in-combinations, i.e., within the context of the run.

This algorithm finds the MPEMP by sequentially reducing the utility of executing ecosystem-damaging actions under the (gradually-weakened) constraint of staying close to the distribution defined by the updated $\boldsymbol{\beta}_{H}^{(G r p)}$. Because the smallest changes in values contained in $\boldsymbol{\beta}_{H}^{(G r p)}$ have been found that achieve the desired ecosystem goals, there are no other group-behavior changes that require smaller changes in group belief systems before such behaviors change enough to achieve the desired ecosystem goals. Hence, the ecosystem management plan that is based on $\boldsymbol{\beta}_{\text {MPEMP }}$ is the most politically feasible.

To implement the MPEMP in the real world, group beliefs that correspond to parameters that have large differences between the updated $\boldsymbol{\beta}_{H}^{(G r p)}$ and $\boldsymbol{\beta}_{\text {MPEMP }}$ need to be changed in the direction of the $\boldsymbol{\beta}_{\text {MPEMP }}$ value. Methods currently used that attempt to change people's perceptions and values (belief systems) include educational programs, workshops, and advertising.

If the needed degree of belief systems change appears to be beyond available resources, less practical ecosystem management plans can be found by minimizing $f_{\text {ecodam }}\left(\boldsymbol{\beta}^{(G r p)}\right)$ over parameters that define groups for which beliefs can be realistically changed and leaving the parameters of all other groups at their (updated) $\boldsymbol{\beta}_{H}^{(G r p)}$ values.

It is possible that the desired ecosystem state values cannot be achieved by the ecosystem ID under any pattern of output actions issued by group IDs. This situation is indicated by $d_{i} \gg 0$ at the last iteration of the algorithm. 


\section{Cheetah management example}

\subsection{Background}

Cheetah preservation is a prominent example of the difficulties surrounding the preservation of a large land mammal whose range extends over several countries. The main threats to cheetah preservation are loss of habitat, cub predation by other carnivores, and being shot to control their predation on livestock (Gros [7], Kelly and Durant [8]). In [8], the authors note that juvenile survival is reduced by lion predation inside wildlife reserves because these reserves are not big enough for cheetah to find areas uninhabited by lions. Over-crowding of reserves in Africa is widespread (see O'Connell-Rodwell et al. [9]) and cheetah do not compete well for space with other carnivores (Kelly and Durant [8]). Although many cheetah are currently existing on commercial land, this coexistence with man's economic activities may not be a secure long-term solution for cheetah.

One solution would be larger reserves that are free of poachers - possibly enclosed with an electric fence. Such a solution was found to be the most viable for keeping elephants from destroying crops in Namibia (see O'Connell-Rodwell et al. [9]). Pelkey et al. [10] also conclude that reserves with regular anti-poaching and anti-logging patrols are the most effective strategy for African wildlife and forest conservation.

A large portion of cheetah range is controlled by Kenya, Tanzania, and Uganda (see Kingdon [11]). Currently, the poverty rates in Kenya, Tanzania, and Uganda are $52 \%, 36 \%$, and $44 \%$, respectively. The adult literacy rates are $(90 \%, 79 \%)$ (males, females) for Kenya, $(85 \%, 69 \%)$ for Tanzania, and $(79 \%, 59 \%)$ for Uganda (World Resources Institute [12]). With close to half of the population living in poverty, many rural Africans in these countries feel that conservation programs put wildlife ahead of their welfare and that large mammals are a threat to their small irrigated patches of ground and their livestock (Gibson [13]). For these reasons, many such individuals are not interested in biodiversity or wildlife conservation.

Gibson [14] finds that the three reasons for poaching are the need for meat, the need for cash from selling animal "trophies," and the protection of livestock. Gibson's analysis suggests that to reduce poaching, policy packages need to be instituted that (a) deliver meat to specific families - not just to the tribal chief, (b) increase the enforcement of laws against the taking of trophies, and (c) improve livestock protection.

\subsection{Finding the MPEMP for east African cheetah}

Say that a cheetah conservation goal is to have an expected cheetah count of 200 individuals in the Kenyan district of Tsavo 50 years hence, i.e., in the year 2058. Say that only rural resident and pastoralist groups are to have their belief systems modified with all other groups having their parameters held at their updated $\boldsymbol{\beta}_{H}^{(\text {Grp })}$ values.

Figure 1 shows the Kenya-portion of the political-ecological data set used to find 


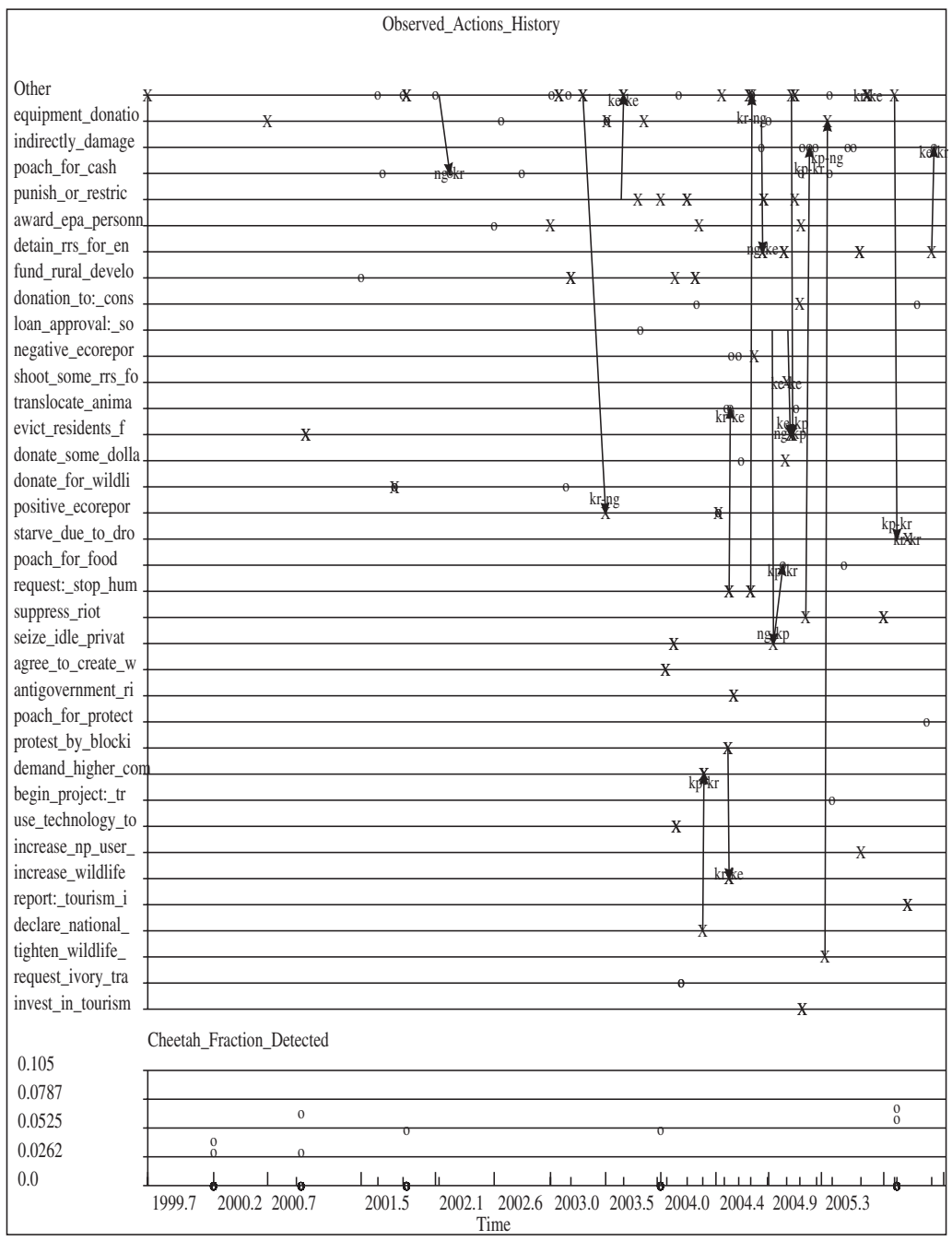

Figure 1: Political-ecological data for Kenya. The ecosystem variable "Cheetah Fraction Detected" is an observable quantity that is derived from the partially unobservable cheetah count, see Haas [4].

the consistent values of the political-ecological process simulator's parameters. In order to show what is predicted to happen if group belief systems remain at their consistent values, Figure 2 shows this consistent model run over the years 2054 through 2058. Figure 3 shows the MPEMP solution over this same time interval. Only the last 5 years are shown because the model quickly enters a repeating pattern of actions and continues this pattern through 2058.

Under the values in the updated $\boldsymbol{\beta}_{H}^{(G r p)}$, the expected cheetah count in the year 2058 for this district is zero but under $\boldsymbol{\beta}_{\text {MPEMP }}$, it is 176. A comparison of Figures 2 


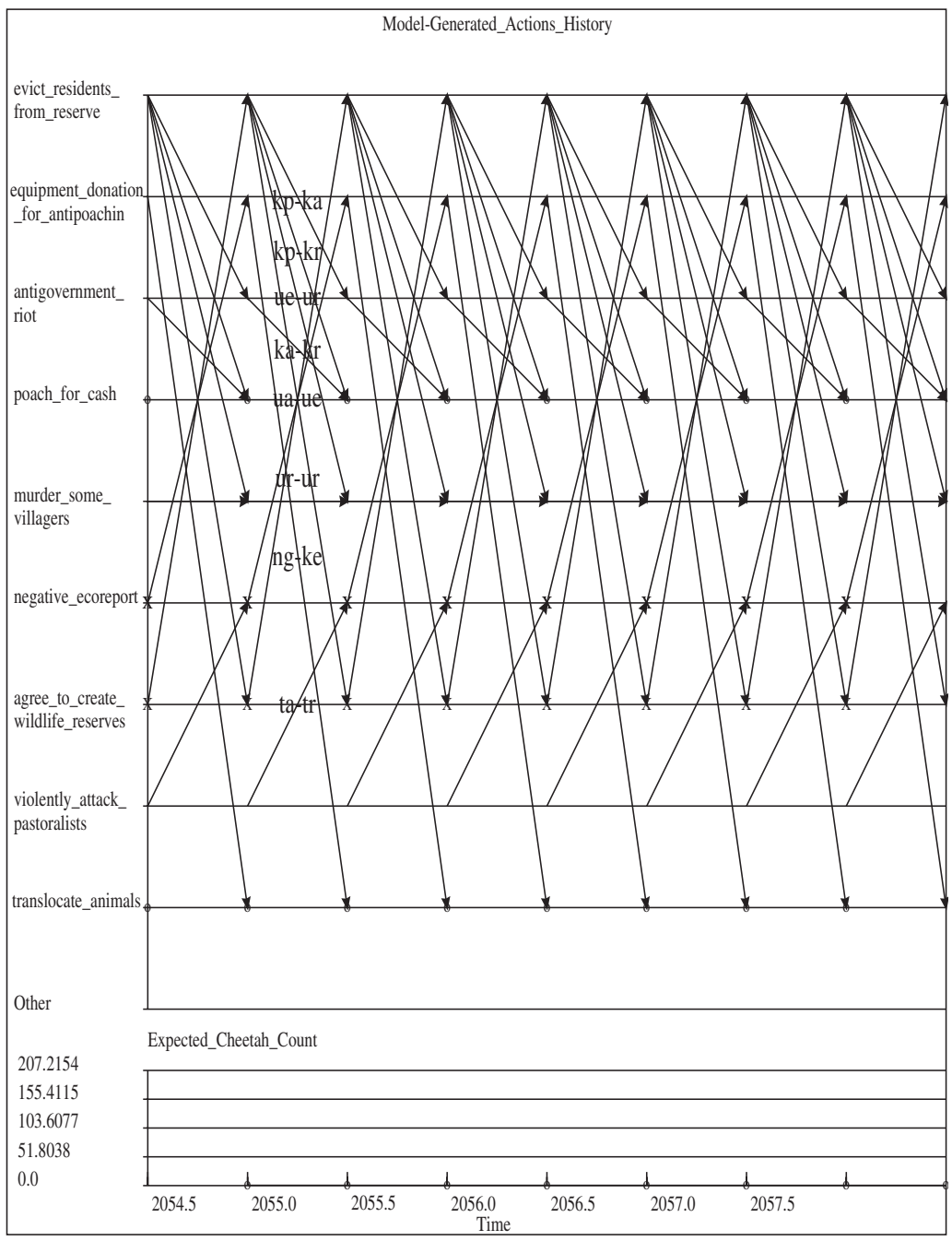

Figure 2: Simulator predictions for 2054-2058 using updated hypothesis parameter values. An arrow's label is located at its center. The first two letters in a label indicates the actor and the second two the target. The first letter in a pair indicates the country (Kenya, Tanzania, or Uganda). The second letter indicates the group type: " $r$ " for rural residents, and "a" for pastoralists. The exception is "ng" for the NGO group.

and 3 reveals that the action: poach for cash is not being executed in the MPEMP scenario. This is because the values contained in the updated $\boldsymbol{\beta}_{H}^{(G r p)}$ that cause this action to be perceived as economically attractive with low risk of arrest are 


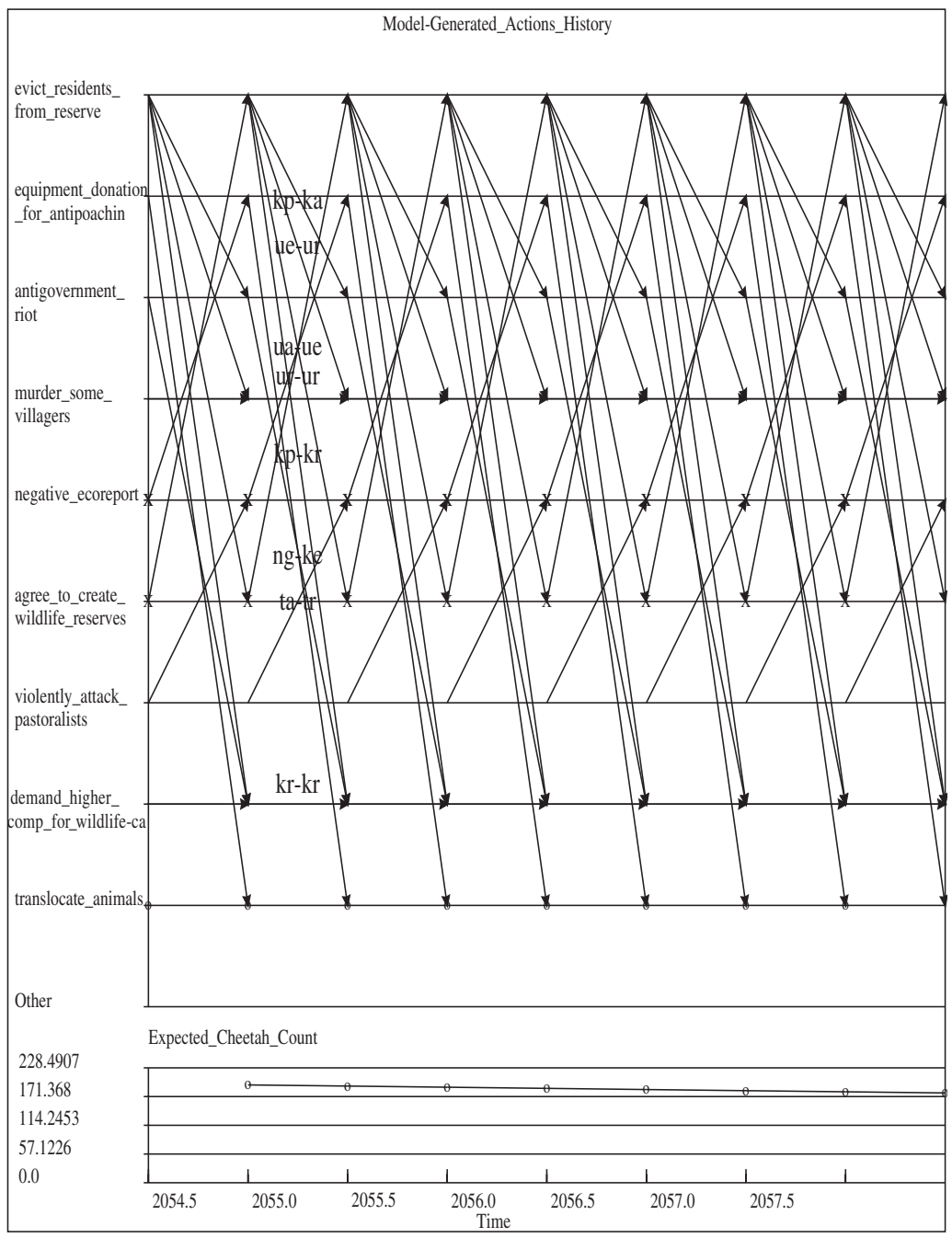

Figure 3: Simulator predictions for 2054-2058 using MPEMP parameter values.

changed to values in $\boldsymbol{\beta}_{\text {MPEMP }}$ that result in the action being perceived as having low economic value and carry a large risk of arrest.

\section{Conclusions}

This modeling, data fitting, and optimization study shows that it is possible to (a) stochastically model the interactions between political and ecological processes, (b) fit this model to political-ecological data, and (c) use this fitted model to find 
the most politically feasible ecosystem management plan under one definition of political feasibility.

All software and data used herein is available at www.uwm.edu/ haas/ cheetah_emt.

\section{References}

[1] Pearl, J., Probabilistic Reasoning in Intelligent Systems, Morgan Kaufmann: San Mateo, CA, p. 125, 1988.

[2] Haas, T.C., Ecosystem management via interacting models of political and ecological processes. Animal Biodiversity and Conservation, 27(1), pp. 231-245, 2004. Available at www.bcn.es/museuciencies.

[3] Haas, T.C., Ecosystem management via interacting models of political and ecological processes. Technical report, Lubar School of Business Administration, University of Wisconsin-Milwaukee, Milwaukee, Wisconsin, 2007.

[4] Haas, T.C., A web-based system for public-private sector collaborative ecosystem management. Stochastic Environmental Research and Risk Assessment, 15(2), pp. 101-131, 2001.

[5] Lindsay, B., Efficiency versus robustness: the case for minimum hellinger distance and related methods. Annals of Statistics, 22, pp. 1081-1114, 1994.

[6] Thompson, J.R. Tapia, R.A., Nonparametric Function Estimation, Modeling and Simulation, Society for Industrial and Applied Mathematics: Philadelphia, p. 179, 1990.

[7] Gros, P.M., Status of the cheetah acinonyx jubatus in kenya: a field-interview assessment. Biological Conservation, 85, pp. 137-149, 1998.

[8] Kelly, M.J. Durant, S.M., Viability of the serengeti cheetah population. Conservation Biology, 14(3), pp. 786-797, 2000.

[9] O’Connell-Rodwell, E.C., Rodwell, T., Matthew, R. Hart, L.A., Living with the modern conservation paradigm: can agricultural communities co-exist with elephants? a five-year case study in east caprivi, namibia. Biological Conservation, 93, pp. 381-391, 2000.

[10] Pelkey, N.W., Stoner, C.J. Caro, T.M., Vegetation in tanzania: assessing long term trends and effects of protection using satellite imagery. Biological Conservation, 94, pp. 297-309, 2000.

[11] Kingdon, J., East African Mammals: An Atlas of Evolution in Africa. Academic Press: London, 1977.

[12] World Resources Institute, Population, Health and Human Well-Being Country Profiles. World Resources Institute: U.K., 2005. Available at http://earthtrends.wri.org.

[13] Gibson, C.C., Politicians and Poachers, Cambridge University Press: Cambridge, U.K., p. 123, 1999.

[14] Gibson, C.C., Politicians and Poachers, p. 122. Gibson [13], 1999. 